\title{
WEAKLY COMPATIBLE MAPS IN FUZZY METRIC SPACES
}

\author{
M. Rangamma, G. Mallikarjun Reddy*, P. Srikanth Rao \\ Department of Mathematics,O.U.,Hyderabad. 500007. \\ *Corresponding address: mrcoolmallik@gmail.com \\ Received 22 October, 2009; Revised 9 July, 2010
}

\begin{abstract}
In this paper, we prove common fixed point theorems for six self maps by using weakly compatibility, without appeal to continuity in fuzzy metric space. Our results extend, generalized several fixed point theorems on metric and fuzzy metric spaces.
\end{abstract}

Mathematics subject classification: $47 \mathrm{H} 10,54 \mathrm{H} 25$.

Keywords : Compatible maps, R-weakly commuting maps, Reciprocal continuity, weakly compatible.

\section{INTRODUCION}

In 1965, the concept of fuzzy sets was intraduced by Zadeh[23].Following the concept of fuzzy sets, fuzzy metric spaces have been intraduced by Kramosil and Michalek[8] and George and Veeramani[3] modified the notion of fuzzy metric spaces with the help of continuous t-norms. Recently many authers have proved fixed point theorems involving fuzzy sets.Vasuki[22] investigated some fixed point theorems in fuzzy metric spaces for R-weakly commuting maps and Pant[15] introduced the notion of reciprocal continuity of mappings in metric spaces.Pant and Jah[17] proved an analogue of result given by Balasubramaniam et al.[1]. S.Kutukcu et.al[12] extended the result of Pant and Jha[17].The aim of this paper is to prove a common fixed point theorem for six mappings by weakly compatibility, without appeal to continuity, which generalize the result of S.kutukcu et.al[12].

\section{Preliminaries}

Definition 1.1: A binary operation $*:[0,1] \times[0,1] \rightarrow[0,1]$ is called a continuous t-norm if $*$ is satisfying the following conditions:
a) * is commutative and associative,
b) $*$ is continuous,
c) $\mathrm{a}^{*} 1=\mathrm{a}$ for all $\mathrm{a} \in[0,1]$,
d) $a^{*} b \leq c^{*} d$ whenever $a \leq c$ and $b \leq d$ for all $a, b, c, d \in[0,1]$.

Definition 1.2 ([3]): A 3-tuple $\left(\mathrm{X}, \mathrm{M},{ }^{*}\right)$ is said to be a fuzzy metric space if $\mathrm{X}$ is an arbitrary set, $*$ is a continuous t-norm and $M$ is a fuzzy set on $X^{2} \times(0, \infty)$ satisfying the following conditions for all $\mathrm{x}, \mathrm{y}, \mathrm{z} \in \mathrm{X}, \mathrm{s}, \mathrm{t}>0$.

(fm1) $\mathrm{M}(\mathrm{x}, \mathrm{y}, \mathrm{t})>0$,

(fm2) $M(x, y, t)=1$ if and only if $x=y$,

$(\mathrm{fm} 3) \mathrm{M}(\mathrm{x}, \mathrm{y}, \mathrm{t})=\mathrm{M}(\mathrm{y}, \mathrm{x}, \mathrm{t})$,

$(\mathrm{fm} 4) \mathrm{M}(\mathrm{x}, \mathrm{y}, \mathrm{t}) * \mathrm{M}(\mathrm{y}, \mathrm{z}, \mathrm{s}) \leq \mathrm{M}(\mathrm{x}, \mathrm{z}, \mathrm{t}+\mathrm{s})$, 
$(f \mathrm{f} 5) \mathrm{M}(\mathrm{x}, \mathrm{y}, \bullet):[0, \infty) \rightarrow[0,1]$ is left continuous .

Then $M$ is called a fuzzy metric on $X$. The function $M(x, y, t)$ denote the degree of nearness between $x$ and $y$ with respect to' $t$ '. We identify $x=y$ with $M(x, y, t)=1$ for all $\mathrm{t}>0$ and $\mathrm{M}(\mathrm{x}, \mathrm{y}, \mathrm{t})=0$ with $\infty$, and we can find some topological properties and examples of fuzzy metric spaces in paper of George and Veeramani[3].

Example 1.3 (Induced fuzzy metric[3]) : Let $(X, d)$ be a metric space.Define $a * b=a b$ for all $a, b \in[0,1]$ and let $M_{d}$ fuzzy sets on $X^{2} \times(0, \infty)$ defined as follows, $M_{d}(x, y, t)=\frac{t}{t+d(x, y)}$ then $\left(X, M_{d}, *\right)$ is a fuzzy metric space. We call this fuzzy metric induced by the metric $\mathrm{d}$, the standard fuzzy metric. On the other hand note that there exists no metric on $\mathrm{X}$ satisfying the above $\mathrm{M}_{\mathrm{d}}(\mathrm{x}, \mathrm{y}, \mathrm{t})$.

Definition 1.4 ([3]): Let (X, $M, *)$ be fuzzy metric space then,

a) A sequence $\left\{x_{n}\right\}$ in $X$ is said to be convergent to $x$ in $X$ if for each $C>0$ and each $t>0$, there exists $n_{0} \in N$ such that $M\left(x_{n}, x, t\right)>1-\epsilon$ for all $n \geq n_{0}$

b) A sequence $\left\{x_{n}\right\}$ in $X$ is said to be Cauchy sequence for each $C>0$ and $t>0$, there exists $\mathrm{n}_{0} \in \mathrm{N}$ such that $\mathrm{M}\left(\mathrm{x}_{\mathrm{m}}, \mathrm{x}_{\mathrm{n}}, \mathrm{t}\right)>1-\mathrm{C}$ for all $\mathrm{m}, \mathrm{n} \geq \mathrm{n}_{0}$.

c) A fuzzy metric space in which every Cauchy sequence is convergent is said to be complete.

Remark 1.5: Since * is continuous, it follows from (fm4) that the limit of the sequence in fuzzy metric space is uniquely determined.

Let $(\mathrm{X}, \mathrm{M}, *)$ is a fuzzy metric space with the following condition (fm6) $\lim _{t \rightarrow \infty} M(x, y, t)=1$ for all $x, y \in X$.

Lemma 1.6([4]): For all $x, y \in X, M(x, y, \bullet)$ is non decreasing.

Lemma 1.7([19]): Let $(X, M, *)$ be a fuzzy metric space if there exists $k \in(0,1)$ such that $M(x, y, k t) \geq M(x, y, t)$ then $x=y$.

Lemma 1.8([2]): Let $\left\{y_{n}\right\}$ be a sequence in a fuzzy metric space $\left(X, M,{ }^{*}\right)$ with the condition (fm6). If there exists a number $\mathrm{k} \in(0,1)$ such that $\mathrm{M}\left(\mathrm{y}_{\mathrm{n}}, \mathrm{y}_{\mathrm{n}+1}, \mathrm{kt}\right) \geq \mathrm{M}\left(\mathrm{y}_{\mathrm{n}-1}, \mathrm{y}_{\mathrm{n}}, \mathrm{t}\right)$ for all $t>0$ and $n \in N$, then $\left\{y_{n}\right\}$ is a Cauchy sequence in $X$.

Proposition 1.9: In a fuzzy metric space $(X, M, *)$, if $a^{*} a \geq a$ for all $a \in[0,1]$ then $a^{*} b=\min \{a, b\}$ for all $a, b \in[0,1]$.

Definition 1.10 ([2]): Two self maps A and $S$ of a fuzzy metric space (X, M, *) are called compatible if $\lim _{n \rightarrow \infty} M\left(A S x_{n}, S A x_{n}, t\right)=1$ whenever $\left\{x_{n}\right\}$ is a sequence in $X$ such that $\lim _{n \rightarrow \infty} A x_{n}=\lim _{n \rightarrow \infty} S x_{n}=x$ for some $x$ in $X$. 
Definition 1.11 ([22]): Two self maps $A$ and $S$ of a fuzzy metric space (X, M, *) are called weakly commuting if $M(A S x, S A x, t) \geq M(A x, S x, t)$ for all $x$ in $X$ and $t>0$.

Definition 1.12 ([22]): Two self maps $A$ and $S$ of a fuzzy metric space (X, M, *) are called R-weakly commuting if there exists $R>0$ such that $M(A S x, S A x, t) \geq M\left(A x, S x, \frac{t}{R}\right)$ for all $\mathrm{x}$ in $\mathrm{X}$ and $\mathrm{t}>0$.

Remark 1.13: Clearly, point wise R-weakly commuting implies weak commuting only when $\mathrm{R} \leq 1$.

Remark 1.14: Compatible mappings are point wise R-weakly commuting but not conversely.

Definition 1.15([1]): Two self maps $A$ and $S$ of a fuzzy metric space $(X, M, *)$ are called reciprocally continuous on $X$ if $\lim _{n \rightarrow \infty} A_{n} x_{n}=A x$ and $\lim _{n \rightarrow \infty} S A x_{n}=S x$ whenever $\left\{x_{n}\right\}$ is a sequence in $X$ such that $\lim _{n \rightarrow \infty} A x_{n}=\lim _{n \rightarrow \infty} S x_{n}=x$ for some $x$ in $X$.

Remark 1.16: If $A$ and $S$ are both continuous, then they are obviously reciprocally continuous but converse is not true. Moreover, in the setting of common fixed point theorems for point wise R-weakly commuting maps satisfying contractive conditions, continuity of one of the mappings $\mathrm{A}$ or $\mathrm{S}$ implies their reciprocally continuity but not conversely.

The following theorem was proved by S. Kutukcu et al.[12]

Theorem 1.17: Let $(X, M, *)$ be complete fuzzy metric space with $a^{*} a \geq a$ for all $a \in[0,1]$ and the condition (fm6). Let (A, S) and ( B, T) be point wise R-weakly commuting pairs of self mappings of $\mathrm{X}$ such that

a) $\mathrm{A}(\mathrm{X}) \subset \mathrm{T}(\mathrm{X}), \mathrm{B}(\mathrm{X}) \subset \mathrm{S}(\mathrm{X})$,

b) There exists $k \in(0,1)$ such that $M(A x, B y, k t) \geq N(x, y, t)$ where

$\mathrm{N}(\mathrm{x}, \mathrm{y}, \mathrm{t})=\mathrm{M}(\mathrm{Sx}, \mathrm{Ax}, \mathrm{t}) * \mathrm{M}(\mathrm{Ty}, \mathrm{By}, \mathrm{t})^{*} \mathrm{M}(\mathrm{Sx}, \mathrm{Ty}, \mathrm{t})^{*} \mathrm{M}(\mathrm{Ty}, \mathrm{Ax}, \alpha \mathrm{t}) * \mathrm{M}(\mathrm{Sx}, \mathrm{By},(2-\alpha) \mathrm{t})$ for all $x, y \in X, \quad \alpha \in(0,2)$ and $t>0$,

If one of the mappings in compatible pair $(\mathrm{A}, \mathrm{S})$ or $(\mathrm{B}, \mathrm{T})$ is continuous then $\mathrm{A}, \mathrm{B}, \mathrm{S}$ and $\mathrm{T}$ have a unique common fixed point.

Definition 1.18([7]): Two self mappings $A$ and $S$ of a fuzzy metric space $(X, M, *)$ are said to be weakly compatible if they commute at their coincidence points that is the pair $(\mathrm{A}, \mathrm{S})$ is weakly compatible pair if $\mathrm{Ax}=\mathrm{Sx}$ implies $\mathrm{ASx}=\mathrm{SAx}$ for all $\mathrm{x} \in \mathrm{X}$.

\section{RESULTS}

In this section we extend the theorem 1.17 by using weakly compatibility which generalize the results of [1], [17] and [12]. 
2.1Compatible mappings are weakly compatible but not converse by using this we extend the theorem 1.17 by implying six weakly compatible maps instead of four compatible mappings in fuzzy metric space.

2.2 Theorem: Let $(X, M, *)$ be a fuzzy metric with $a^{*} a \geq$ a for all $a \in[0,1]$ and the condition (fm6). Let $\mathrm{A}, \mathrm{B}, \mathrm{S}, \mathrm{T}, \mathrm{P}$ and $\mathrm{Q}$ be mappings from $\mathrm{X}$ into itself such that

(2.2.1) $\mathrm{P}(\mathrm{X}) \subset \mathrm{AB}(\mathrm{X}), \mathrm{Q}(\mathrm{X}) \subset \mathrm{ST}(\mathrm{X})$,

(2.2.2) The pairs $(\mathrm{A}, \mathrm{B}),(\mathrm{S}, \mathrm{T}),(\mathrm{P}, \mathrm{S}),(\mathrm{Q}, \mathrm{B}),(\mathrm{T}, \mathrm{P})$ and $(\mathrm{A}, \mathrm{Q})$ are commuting mappings

(2.2.3) The pairs $(\mathrm{P}, \mathrm{ST})$ and $(\mathrm{Q}, \mathrm{AB})$ are weakly compatible.

(2.2.4) There exists a number $k \in(0,1)$ such that $M(P x, Q y, k t) \geq N(x, y, t)$ where $N(x, y, t)=M(S T x, P x, t) * M(A B y, Q y, t) * M(S T x, A B y, t)$

$* \mathrm{M}(\mathrm{ABy}, \mathrm{Px}, \alpha \mathrm{t}) * \mathrm{M}(\mathrm{STx}, \mathrm{Qy},(2-\alpha) \mathrm{t})$

for all $\mathrm{x}, \mathrm{y} \in \mathrm{X}, \alpha \in(0,2)$ and $\mathrm{t}>0$, then $\mathrm{A}, \mathrm{S}, \mathrm{P}, \mathrm{Q}, \mathrm{T}$ and $\mathrm{B}$ have a unique common fixed point in $\mathrm{X}$.

Proof: $B y(2.2 .1) P(X) \subset A B(X)$, for any $x_{0} \in X$ there exists a point $x_{1} \in X$ such that $\mathrm{Px}_{0}=\mathrm{ABx}_{1}$

Since $\mathrm{Q}(\mathrm{X}) \subset \mathrm{ST}(\mathrm{X})$ for this point $\mathrm{x}_{1}$, we choose a point $\mathrm{x}_{2} \in \mathrm{X}$ such that $\mathrm{Qx}_{1}=\mathrm{STx}_{2}$ Inductively, we can find a sequence $\left\{\mathrm{y}_{\mathrm{n}}\right\}$ in $\mathrm{X}$ as follows

(2.2.5) $\mathrm{y}_{2 \mathrm{n}}=\mathrm{Px}_{2 \mathrm{n}}=\mathrm{ABx}_{2 \mathrm{n}+1}$ and $\mathrm{y}_{2 \mathrm{n}+1}=\mathrm{Qx}_{2 \mathrm{n}+1}=\mathrm{STx}_{2 \mathrm{n}+2}$ for $\mathrm{n}=0,1, \ldots$

$\operatorname{By}(2.2 .4)$ for all $\mathrm{t}>0$ and $\alpha=1-\mathrm{q}$ with $\mathrm{q} \in(0,1)$, we have

(2.2.6) $\mathrm{M}\left(\mathrm{Px}_{2 \mathrm{n}}, \mathrm{Qx}_{2 \mathrm{n}+1}, \mathrm{kt}\right) \geq \mathrm{M}\left(\mathrm{ST}_{2 \mathrm{n}}, \mathrm{Px}_{2 \mathrm{n}}, \mathrm{t}\right) * \mathrm{M}\left(\mathrm{ABx}_{2 \mathrm{n}+1}, \mathrm{Qx}_{2 \mathrm{n}+1}, \mathrm{t}\right) * \mathrm{M}\left(\mathrm{Tx}_{2 \mathrm{n}}, \mathrm{ABx}_{2 \mathrm{n}+1}, \mathrm{t}\right)^{*}$ $\mathrm{M}\left(\mathrm{ABx}_{2 \mathrm{n}+1}, \mathrm{Px}_{2 \mathrm{n}},(1-\mathrm{q}) \mathrm{t}\right)^{*} \mathrm{M}\left(\mathrm{STx}_{2 \mathrm{n}}, \mathrm{Qx}_{2 \mathrm{n}+1},(1+\mathrm{q}) \mathrm{t}\right)$

(2.2.7) $M\left(y_{n}, y_{2 n+1}, k t\right) \geq M\left(y_{2 n-1}, y_{2 n}, t\right)^{*} M\left(y_{2 n}, y_{2 n+1}, t\right) * M\left(y_{2 n-1}, y_{2 n}, t\right) * M\left(y_{2 n}, y_{2 n},(1-q) t\right)^{*}$ $\mathrm{M}\left(\mathrm{y}_{2 \mathrm{n}-1}, \mathrm{y}_{2 \mathrm{n}+1},(1+\mathrm{q}) \mathrm{t}\right)$ $\geq M\left(y_{2 n-1}, y_{2 n}, t\right) * M\left(y_{2 n}, y_{2 n+1}, t\right) * M\left(y_{2 n-1}, y_{2 n}, t\right) * M\left(y_{2 n}, y_{2 n+1}, q t\right)$

Since t-norm $*$ is continuous, letting $\mathrm{q} \rightarrow 1$, we have

It follows that

$$
\begin{aligned}
& \geq M\left(y_{2 n-1}, y_{2 n}, t\right)^{*} M\left(y_{2 n}, y_{2 n+1}, t\right) * M\left(y_{2 n}, y_{2 n+1}, t\right) \\
& \geq M\left(y_{2 n-1}, y_{2 n}, t\right)^{*} M\left(y_{2 n}, y_{2 n+1}, t\right)
\end{aligned}
$$

(2.2.8) $M\left(y_{2 n}, y_{2 n+1}, k t\right) \geq M\left(y_{2 n-1}, y_{2 n}, t\right)^{*} M\left(y_{2 n}, y_{2 n+1}, t\right)$.

Similarly

(2.2.9) $M\left(y_{2 n+1}, y_{2 n+2}, k t\right) \geq M\left(y_{2 n}, y_{2 n+1}, t\right) * M\left(y_{2 n+1}, y_{2 n+2}, t\right)$.

Therefore for all $\mathrm{n}$ even and odd, we have

(2.2.10) $M\left(y_{n}, y_{n+1}, k t\right) \geq M\left(y_{n-1}, y_{n}, t\right)^{*} M\left(y_{n}, y_{n+1}, t\right)$

Consequently

(2.2.11) $M\left(y_{n}, y_{n+1}, t\right) \geq M\left(y_{n-1}, y_{n}, k^{-1} t\right) * M\left(y_{n}, y_{n+1}, k^{-1} t\right)$

Repeated application of this inequality will imply that

$M\left(y_{n}, y_{n+1}, t\right) \geq M\left(y_{n-1}, y_{n}, k^{-1} t\right) * M\left(y_{n}, y_{n+1}, k^{-1} t\right) \geq \ldots .$.

$\ldots . . \geq M\left(y_{n-1}, y_{n}, k^{-1} t\right)^{*} M\left(y_{n}, y_{n+1}, k^{-m_{n}} t\right)$ where $m \in N$

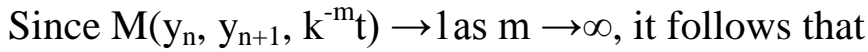


(2.2.12) $M\left(y_{n}, y_{n+1}, t\right) \geq M\left(y_{n-1}, y_{n}, k^{-1} t\right)$ for all $n \in N$

Consequently

$\mathrm{M}\left(\mathrm{y}_{\mathrm{n}}, \mathrm{y}_{\mathrm{n}+1}, \mathrm{kt}\right) \geq \mathrm{M}\left(\mathrm{y}_{\mathrm{n}-1}, \mathrm{y}_{\mathrm{n}}, \mathrm{t}\right)$ for all $\mathrm{n} \in \mathrm{N}$

Therefore, by the lemma 1.8, $\left\{y_{n}\right\}$ is Cauchy Sequence in $X$. Since $X$ is complete $\left\{y_{n}\right\}$ converges to a point $z \in X$. Since $\left\{\mathrm{Px}_{2 \mathrm{n}}\right\},\left\{\mathrm{Qx}_{2 \mathrm{n}+1}\right\},\left\{\mathrm{ABx}_{2 \mathrm{n}+1}\right\}$ and $\left\{\mathrm{STx}_{2 \mathrm{n}+2}\right\}$ are sub sequences of $\left\{y_{n}\right\}$, they also converge to the point $\mathrm{z}$, that is

(2.2.13) $\mathrm{Px}_{2 \mathrm{n}}, \mathrm{Qx}_{2 \mathrm{n}+1}, \mathrm{ABx}_{2 \mathrm{n}+2}, \mathrm{STx}_{2 \mathrm{n}+2} \rightarrow \mathrm{z}$ as $\mathrm{n} \rightarrow \infty$.

Since $\mathrm{P}(\mathrm{X}) \subset \mathrm{AB}(\mathrm{X})$, for a point $\mathrm{u} \in \mathrm{X}$ such that $\mathrm{ABu}=\mathrm{z}$

Since $\mathrm{Q}(\mathrm{X}) \subset \mathrm{ST}(\mathrm{X})$, for a point $v \in X$ such that $\mathrm{ST} v=\mathrm{z}$

Putting $\mathrm{x}=\mathrm{v}, \mathrm{y}=\mathrm{x}_{2 \mathrm{n}+1}$ with $\alpha=1$ in (2.2.4)

$(2.2 .14) \mathrm{M}\left(\mathrm{Pv}, \mathrm{Qx}_{2 \mathrm{n}+1}, \mathrm{kt}\right) \geq \mathrm{N}\left(\mathrm{v}, \mathrm{x}_{2 \mathrm{n}+1}, \mathrm{t}\right)$

$$
\begin{aligned}
& \geq \mathrm{M}(\mathrm{STv}, \mathrm{Pv}, \mathrm{t})^{*} \mathrm{M}\left(\mathrm{ABx}_{2 \mathrm{n}+1}, \mathrm{Qx}_{2 \mathrm{n}+1}, \mathrm{t}\right)^{*} \mathrm{M}\left(\mathrm{STv}, \mathrm{ABx}_{2 \mathrm{n}+1}, \mathrm{t}\right) \\
& \quad * \mathrm{M}\left(\mathrm{ABx}_{2 \mathrm{n}+1}, \mathrm{Pv}, \mathrm{t}\right)^{*} \mathrm{M}\left(\mathrm{STv}, \mathrm{Qx}_{2 \mathrm{n}+1}, \mathrm{t}\right)
\end{aligned}
$$

Proceeding limit as $\mathrm{n} \rightarrow \infty$, we have

(2.2.15)M(Pv, z, kt) $\geq \mathrm{M}(\mathrm{z}, \mathrm{Pv}, \mathrm{t}) * \mathrm{M}(\mathrm{z}, \mathrm{z}, \mathrm{t}) * \mathrm{M}(\mathrm{z}, \mathrm{z}, \mathrm{t})^{*} \mathrm{M}(\mathrm{z}, \mathrm{Pv}, \mathrm{t})^{*} \mathrm{M}(\mathrm{z}, \mathrm{z}, \mathrm{t})$

$$
\geq \mathrm{M}(\mathrm{z}, \mathrm{Pv}, \mathrm{t})
$$

which gives $\mathrm{Pv}=\mathrm{z}$, therefore

(2.2.16) $\mathrm{STv}=\mathrm{Pv}=\mathrm{z}$

$\mathrm{P}, \mathrm{ST}$ are weakly compatible, so they commute at coincidence point therefore

$\mathrm{P}(\mathrm{STv})=(\mathrm{ST}) \mathrm{Pv}$ that is $\mathrm{Pz}=\mathrm{STz}$ thus

(2.2.17) $\mathrm{Pz}=\mathrm{STz}$

Putting $\mathrm{x}=\mathrm{v}, \mathrm{y}=\mathrm{u}$ with $\alpha=1$ in (2.2.4)

(2.2.18) $\mathrm{M}(\mathrm{Pv}, \mathrm{Qu}, \mathrm{kt}) \geq \mathrm{N}(\mathrm{v}, \mathrm{u}, \mathrm{t})$ $\geq \mathrm{M}(\mathrm{STv}, \mathrm{Pv}, \mathrm{t}) * \mathrm{M}(\mathrm{ABu}, \mathrm{Qu}, \mathrm{t}) * \mathrm{M}(\mathrm{ST} v, \mathrm{ABu}, \mathrm{t}) * \mathrm{M}(\mathrm{ABu}, \mathrm{Pv}, \mathrm{t})$

$* \mathrm{M}(\mathrm{ST} v, \mathrm{Qu}, \mathrm{t})$

$\geq \mathrm{M}(\mathrm{z}, \mathrm{z}, \mathrm{t}) * \mathrm{M}(\mathrm{z}, \mathrm{Qu}, \mathrm{t})^{*} \mathrm{M}(\mathrm{z}, \mathrm{z}, \mathrm{t})^{*} \mathrm{M}(\mathrm{z}, \mathrm{z}, \mathrm{t}) * \mathrm{M}(\mathrm{z}, \mathrm{Qu}, \mathrm{t})$,

which gives $\mathrm{z}=\mathrm{Qu}$.

Therefore $\mathrm{Qu}=\mathrm{z}=\mathrm{ABu}$

since $(\mathrm{Q}, \mathrm{AB})$ is weakly compatible pair $(\mathrm{AB}) \mathrm{Qu}=\mathrm{Q}(\mathrm{ABu})$ implies $\mathrm{ABz}=\mathrm{Qz}$ Thus

(2.2.19) $\mathrm{ABz}=\mathrm{Qz}$

Now we show that $\mathrm{z}$ is the fixed point of $\mathrm{Q}$, so by putting $\mathrm{x}=\mathrm{x}_{2 \mathrm{n}}, \mathrm{y}=\mathrm{z}$ with $\alpha=1$ in

(2.2.4) we have

$(2.2 .20) \mathrm{M}\left(\mathrm{Px}_{2 \mathrm{n}}, \mathrm{Qz}, \mathrm{kt}\right) \geq \mathrm{N}\left(\mathrm{x}_{2 \mathrm{n}}, \mathrm{z}, \mathrm{t}\right)$

$$
\begin{aligned}
& \geq \mathrm{M}\left(\mathrm{STx}_{2 \mathrm{n}}, \mathrm{Px}_{2 \mathrm{n}}, \mathrm{t}\right) * \mathrm{M}(\mathrm{ABz}, \mathrm{Qz}, \mathrm{t}) * \mathrm{M}\left(\mathrm{STx}_{2 \mathrm{n}}, \mathrm{ABz}, \mathrm{t}\right) \\
& * \mathrm{M}\left(\mathrm{ABz}, \mathrm{Px}_{2 \mathrm{n}}, \mathrm{t}\right) * \mathrm{M}\left(\mathrm{STx}_{2 \mathrm{n}}, \mathrm{Qz}, \mathrm{t}\right)
\end{aligned}
$$

letting $\mathrm{n} \rightarrow \infty$

$$
\geq \mathrm{M}(\mathrm{z}, \mathrm{z}, \mathrm{t}) * \mathrm{M}(\mathrm{Qz}, \mathrm{Qz}, \mathrm{t}) * \mathrm{M}(\mathrm{z}, \mathrm{Qz}, \mathrm{t}) * \mathrm{M}(\mathrm{Qz}, \mathrm{z}, \mathrm{t}) * \mathrm{M}(\mathrm{z}, \mathrm{Qz}, \mathrm{t})
$$


\[ \begin{array}{l}\geq \mathrm{M}(\mathrm{z}, \mathrm{Qz}, \mathrm{t}) \\ \text { which shows } \mathrm{z}=\mathrm{Qz}\end{array} \]

(2.2.21)thus $\mathrm{z}=\mathrm{Qz}=\mathrm{ABz}$

Now, we show that $\mathrm{z}$ is the fixed point of $\mathrm{P}$ by putting $\mathrm{x}=\mathrm{z}, \mathrm{y}=\mathrm{x}_{2 \mathrm{n}+1}$ with $\alpha=1$ in (2.2.4) we have

(2.2.22) $\mathrm{M}\left(\mathrm{Pz}, \mathrm{Qx}_{2 \mathrm{n}+1}, \mathrm{kt}\right) \geq \mathrm{N}\left(\mathrm{z}, \mathrm{x}_{2 \mathrm{n}+1}, \mathrm{t}\right)$

letting $\mathrm{n} \rightarrow \infty$

$$
\begin{aligned}
& \geq \mathrm{M}(\mathrm{STz}, \mathrm{Pz}, \mathrm{t}) * \mathrm{M}\left(\mathrm{ABx}_{2 \mathrm{n}+1}, \mathrm{Qx}_{2 \mathrm{n}+1}, \mathrm{t}\right)^{*} \mathrm{M}\left(\mathrm{STz}, \mathrm{ABx}_{2 \mathrm{n}+1}, \mathrm{t}\right) \\
& \quad * \mathrm{M}\left(\mathrm{ABx}_{2 \mathrm{n}+1}, \mathrm{Pz}, \mathrm{t}\right)^{*} \mathrm{M}\left(\mathrm{STz}, \mathrm{Qx}_{2 \mathrm{n}+1}, \mathrm{t}\right)
\end{aligned}
$$

$\mathrm{M}(\mathrm{Pz}, \mathrm{z}, \mathrm{kt}) \geq \mathrm{M}(\mathrm{Pz}, \mathrm{Pz}, \mathrm{t}) * \mathrm{M}(\mathrm{z}, \mathrm{z}, \mathrm{t}) * \mathrm{M}(\mathrm{Pz}, \mathrm{z}, \mathrm{t}) * \mathrm{M}(\mathrm{z}, \mathrm{Pz}, \mathrm{t}) * \mathrm{M}(\mathrm{Pz}, \mathrm{z} \mathrm{t})$

$$
\geq \mathrm{M}(\mathrm{z}, \mathrm{Pz}, \mathrm{t})
$$

which shows $\mathrm{z}=\mathrm{Pz}$

(2.2.23)Thus $\mathrm{Pz}=\mathrm{z}=\mathrm{STz}$.

Now, we show that $\mathrm{z}=\mathrm{Tz}$, by putting $\mathrm{x}=\mathrm{Tz}$ and $\mathrm{y}=\mathrm{x}_{2 \mathrm{n}+1}$ with $\alpha=1$ in (2.2.4) and using (2.2.2)

(2.2.24)M(P(Tz), $\left.\mathrm{Qx}_{2 \mathrm{n}+1}, \mathrm{kt}\right) \geq \mathrm{N}\left(\mathrm{Tz}, \mathrm{x}_{2 \mathrm{n}+1}, \mathrm{t}\right)$

$$
\begin{aligned}
& \geq \mathrm{M}(\mathrm{ST}(\mathrm{Tz}), \mathrm{P}(\mathrm{Tz}), \mathrm{t}) * \mathrm{M}\left(\mathrm{ABx}_{2 \mathrm{n}+1}, \mathrm{Qx}_{2 \mathrm{n}+1}, \mathrm{t}\right) * \mathrm{M}\left(\mathrm{ST}(\mathrm{Tz}), \mathrm{ABx}_{2 \mathrm{n}+1}, \mathrm{t}\right) \\
& * \mathrm{M}\left(\mathrm{ABx}_{2 \mathrm{n}+1}, \mathrm{P}(\mathrm{Tz}), \mathrm{t}\right) * \mathrm{M}\left(\mathrm{ST}(\mathrm{Tz}), \mathrm{Qx}_{2 \mathrm{n}+1}, \mathrm{t}\right)
\end{aligned}
$$

letting $\mathrm{n} \rightarrow \infty$ and using (2.2.23)

$(2.2 .25) \mathrm{M}(\mathrm{Tz}, \mathrm{z}, \mathrm{kt}) \geq \mathrm{M}(\mathrm{Tz}, \mathrm{Tz}, \mathrm{t}) * \mathrm{M}(\mathrm{z}, \mathrm{z}, \mathrm{t}) * \mathrm{M}(\mathrm{Tz}, \mathrm{z}, \mathrm{t}) * \mathrm{M}(\mathrm{z}, \mathrm{Tz}, \mathrm{t}) * \mathrm{M}(\mathrm{Tz}, \mathrm{z}, \mathrm{t})$

$$
\geq \mathrm{M}(\mathrm{Tz}, \mathrm{z}, \mathrm{t})
$$

which gives $\mathrm{z}=\mathrm{Tz}$.

Since $\mathrm{STz}=\mathrm{z}$ gives $\mathrm{Sz}=\mathrm{z}$,

Finally we have to show that $\mathrm{Bz}=\mathrm{z}$.

By putting $\mathrm{x}=\mathrm{z}, \mathrm{y}=\mathrm{Bz}$ with $\alpha=1$ in (2.2.4) and using (2.2.2)

(2.2.26) $\mathrm{M}(\mathrm{Pz}, \mathrm{QBz}, \mathrm{kt}) \geq \mathrm{N}(\mathrm{z}, \mathrm{Bz}, \mathrm{t})$

$$
\begin{aligned}
& \geq \mathrm{M}(\mathrm{STz}, \mathrm{Pz}, \mathrm{t}) * \mathrm{M}(\mathrm{AB}(\mathrm{Bz}), \mathrm{Q}(\mathrm{Bz}), \mathrm{t}) * \mathrm{M}(\mathrm{STz}, \mathrm{AB}(\mathrm{Bz}), \mathrm{t}) \\
& \quad \mathrm{M}(\mathrm{AB}(\mathrm{Bz}), \mathrm{Pz}, \mathrm{t}) * \mathrm{M}(\mathrm{STz}, \mathrm{Q}(\mathrm{Bz}), \mathrm{t}) \\
& \geq \mathrm{M}(\mathrm{z}, \mathrm{z}, \mathrm{t}) * \mathrm{M}(\mathrm{Bz}, \mathrm{Bz}, \mathrm{t}) * \mathrm{M}(\mathrm{z}, \mathrm{Bz}, \mathrm{t}) * \mathrm{M}(\mathrm{Bz}, \mathrm{z}, \mathrm{t}) * \mathrm{M}(\mathrm{z}, \mathrm{Bz}, \mathrm{t})
\end{aligned}
$$

$\mathrm{M}(\mathrm{z}, \mathrm{Bz}, \mathrm{t}) \geq \mathrm{M}(\mathrm{z}, \mathrm{Bz}, \mathrm{t})$

which gives $\mathrm{z}=\mathrm{Bz}$.

Since $\mathrm{ABz}=\mathrm{z}$ implies $\mathrm{Az}=\mathrm{z}$

By combining the above results, we have

(2.2.27) $\mathrm{Az}=\mathrm{Bz}=\mathrm{Sz}=\mathrm{Tz}=\mathrm{Pz}=\mathrm{Qz}=\mathrm{z}$

That is $z$ is the common fixed point of $A, B, S, T$, Pand Q. For uniqueness, let $\mathrm{w}(\mathrm{w} \neq \mathrm{z})$ be another common fixed point of $\mathrm{A}, \mathrm{B}, \mathrm{S}, \mathrm{T}, \mathrm{P}$ and $\mathrm{Q}$ with $\alpha=1$ then by (2.2.4), we write

(2.2.28) $\mathrm{M}(\mathrm{Pz}, \mathrm{Qw}, \mathrm{kt}) \geq \mathrm{M}(\mathrm{STz}, \mathrm{Pz}, \mathrm{t}) * \mathrm{M}(\mathrm{ABw}, \mathrm{Qw}, \mathrm{t}) * \mathrm{M}(\mathrm{STz}, \mathrm{ABw}, \mathrm{t}) * \mathrm{M}(\mathrm{ABw}, \mathrm{Pz}, \mathrm{t})$ 


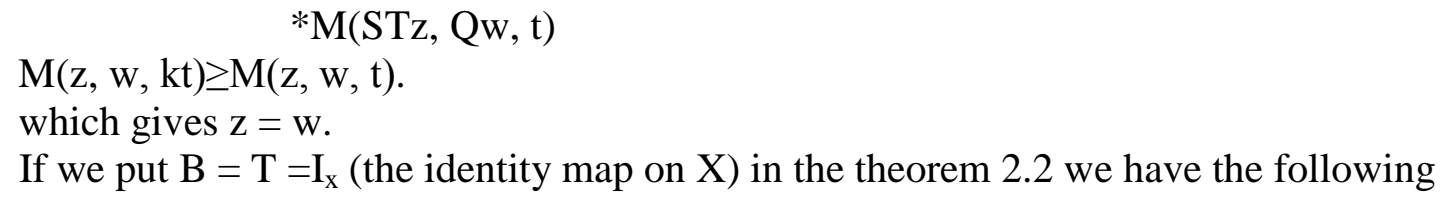

Corollary (2.3): Let $\left(\mathrm{X}, \mathrm{M},{ }^{*}\right)$ be a complete fuzzy metric space with $\mathrm{a}^{*} \mathrm{a} \geq \mathrm{a}$ for all $\mathrm{a} \in[0,1]$ and the condition (fm6).

Let A, S, P, Q be mappings from X into itself such that

(2.3.1) $\mathrm{P}(\mathrm{X}) \subset \mathrm{A}(\mathrm{X}), \mathrm{Q}(\mathrm{X}) \subset \mathrm{S}(\mathrm{X})$,

(2.3.2) The pair $(\mathrm{P}, \mathrm{S})$ and $(\mathrm{Q}, \mathrm{A})$ are weakly compatible,

(2.3.3) There exists a number $\mathrm{k} \in(0,1)$ such that $\mathrm{M}(\mathrm{Px}, \mathrm{Qy}, \mathrm{kt}) \geq \mathrm{M}(\mathrm{Sx}, \mathrm{Px}, \mathrm{t}){ }^{*} \mathrm{M}(\mathrm{Ay}, \mathrm{Qy}, \mathrm{t}) * \mathrm{M}(\mathrm{Sx}, \mathrm{Ay}, \mathrm{t}) * \mathrm{M}(\mathrm{Ay}, \mathrm{Px}, \alpha \mathrm{t})$ $* \mathrm{M}(\mathrm{Sx}, \mathrm{Qy},(2-\alpha) \mathrm{t})$

for all $\mathrm{x}, \mathrm{y} \in \mathrm{X}, \alpha \in(0,2)$ and $\mathrm{t}>0$ then $\mathrm{A}, \mathrm{S}, \mathrm{P}$ and $\mathrm{Q}$ have a unique common fixed point in $\mathrm{X}$.

If we put $\mathrm{P}=\mathrm{Q}, \mathrm{B}=\mathrm{T}=\mathrm{I}_{\mathrm{x}}$ in theorem 2.2 we have the following

Corollary (2.4): Let $\left(X, M,{ }^{*}\right)$ be a complete fuzzy metric space with $a * a \geq a$, for all $a \in[0,1]$ and the condition (fm6).Let A, S, T be mappings from $\mathrm{X}$ into itself such that

(2.4.1) $\mathrm{P}(\mathrm{X}) \subset \mathrm{A}(\mathrm{X}), \mathrm{P}(\mathrm{X}) \subset \mathrm{S}(\mathrm{X})$,

(2.4.2) The pairs $(\mathrm{P}, \mathrm{A})$ and $(\mathrm{P}, \mathrm{S})$ are weakly compatible,

(2.4.3) There exists a number $\mathrm{k} \in(0,1)$ such that

$\mathrm{M}(\mathrm{Px}, \mathrm{Py}, \mathrm{kt}) \geq \mathrm{N}(\mathrm{x}, \mathrm{y}, \mathrm{t})$

where $N(x, y, t)=M(S x, P x, t) * M(A y, P y, t) * M(S x, A y, t) * M(A y, P x, \alpha t)$ $* \mathrm{M}(\mathrm{Sx}, \mathrm{Py},(2-\alpha) \mathrm{t})$

for all $\mathrm{x}, \mathrm{y} \in \mathrm{X}, \alpha \in(0,2)$ and $\mathrm{t}>0$ then $\mathrm{P}, \mathrm{S}, \mathrm{A}$ have a unique common fixed point.

If we put $\mathrm{P}=\mathrm{Q}, \mathrm{A}=\mathrm{S}$ and $\mathrm{B}=\mathrm{T}=\mathrm{I}_{\mathrm{x}}$ in theorem 2.2 we have the following

Corollary (2.5): Let $(X, M, *)$ be complete fuzzy metric space with $a^{*} a \geq a$ for all $a \in[0,1]$ and the condition (fm6). Let $\mathrm{P}, \mathrm{S}$ be weakly compatible pair of self maps such that, $\mathrm{P}(\mathrm{X}) \subset \mathrm{S}(\mathrm{X})$ and there exists a constant $\mathrm{k} \in(0,1)$ such that $\mathrm{M}(\mathrm{Px}, \mathrm{Py}, \mathrm{kt}) \geq \mathrm{M}(\mathrm{Sx}, \mathrm{Px}, \mathrm{t}) * \mathrm{M}(\mathrm{Sy}, \mathrm{Py}, \mathrm{t}) * \mathrm{M}(\mathrm{Sx}, \mathrm{Sy}, \mathrm{t}) * \mathrm{M}(\mathrm{Sy}, \mathrm{Px}, \alpha \mathrm{t})$ $* \mathrm{M}(\mathrm{Sx}, \mathrm{Py},(2-\alpha) \mathrm{t})$

for all $\mathrm{x}, \mathrm{y} \in \mathrm{X}, \alpha \in(0,2)$ and $\mathrm{t}>0$, then $\mathrm{P}$ and $\mathrm{S}$ have a unique common fixed point in $X$.

If we put $\mathrm{A}=\mathrm{S}$ and $\mathrm{B}=\mathrm{T}=\mathrm{I}_{\mathrm{x}}$ in theorem 2.2 we have the following.

Corollary (2.6): Let $(X, M, *)$ be complete fuzzy metric space with $a^{*} a \geq a$ for all $a \in[0,1]$ and the condition (fm6). Let $P, Q, S$ be mappings from $X$ to itself such that, 
(2.6.1) $\mathrm{P}(\mathrm{X}) \subset \mathrm{S}(\mathrm{X}), \mathrm{Q}(\mathrm{X}) \subset \mathrm{S}(\mathrm{X})$

(2.6.2) Either $(\mathrm{P}, \mathrm{S})$ or $(\mathrm{Q}, \mathrm{S})$ is weakly compatible pair

(2.6.3) $\mathrm{M}(\mathrm{Px}, \mathrm{Qy}, \mathrm{kt}) \geq \mathrm{M}(\mathrm{Sx}, \mathrm{Px}, \mathrm{t}) * \mathrm{M}(\mathrm{Sy}, \mathrm{Qy}, \mathrm{t}) * \mathrm{M}(\mathrm{Sy}, \mathrm{Sy}, \mathrm{t}) * \mathrm{M}(\mathrm{Sy}, \mathrm{Px}, \alpha \mathrm{t})$ $* \mathrm{M}(\mathrm{Sx}, \mathrm{Qy},(2-\alpha) \mathrm{t})$

for all $\mathrm{x}, \mathrm{y} \in \mathrm{X}, \alpha \in(0,2)$ and $\mathrm{t}>0$

then $\mathrm{P}, \mathrm{Q}$ and $\mathrm{S}$ have a unique common fixed point in $\mathrm{X}$.

Remark (2.7): Since $\mathrm{a}^{*} \mathrm{~b}=\min \{\mathrm{a}, \mathrm{b}\}$ then the condition (2.2.4) in the theorem 2.2 becomes $\mathrm{M}(\mathrm{Px}, \mathrm{Qy}, \mathrm{kt}) \geq \min \{\mathrm{M}(\mathrm{ST} x, \mathrm{Px}, \mathrm{t}) * \mathrm{M}(\mathrm{ABy}, \mathrm{Qy}, \mathrm{t}) * \mathrm{M}(\mathrm{ST} x, \mathrm{ABy}, \mathrm{t})$

$$
* \mathrm{M}(\mathrm{ABy}, \mathrm{Px}, \alpha \mathrm{t}) * \mathrm{M}(\mathrm{STx}, \mathrm{Qy},(2-\alpha) \mathrm{t})\}
$$

for all $\mathrm{x}, \mathrm{y} \in \mathrm{X}, \alpha \in(0,2)$ and $\mathrm{t}>0$

Now, we prove the theorem 2.2 from the compatible fuzzy metric space to complete metric space.

Theorem 2.8: Let A, B, S, T, P and Q be self mappings of a complete metric(X, d). Suppose that the pairs $(\mathrm{P}, \mathrm{ST})$ and $(\mathrm{Q}, \mathrm{AB})$ are weakly compatible and also(A, B), $(\mathrm{S}, \mathrm{T})$, $(\mathrm{P}, \mathrm{S}),(\mathrm{A}, \mathrm{Q}),(\mathrm{T}, \mathrm{P}),(\mathrm{Q}, \mathrm{B})$ are commuting mappings with one of $\mathrm{P}, \mathrm{Q}, \mathrm{AB}, \mathrm{ST}$ are continuous. If there exists a constant $\mathrm{k} \in(0,1)$ such that for all $\mathrm{x}, \mathrm{y} \in \mathrm{X}$ $\mathrm{d}(\mathrm{Px}, \mathrm{Qy}) \leq \mathrm{k} \max \{\mathrm{d}(\mathrm{STx}, \mathrm{Px}), \mathrm{d}(\mathrm{ABy}, \mathrm{Qy}), \mathrm{d}(\mathrm{STx}, \mathrm{ABy})$, $\left.\frac{1}{2}[\mathrm{~d}(\mathrm{ABy}, \mathrm{Px})+\mathrm{d}(\mathrm{STx}, \mathrm{Qy})]\right\}$ Then $\mathrm{A}, \mathrm{B}, \mathrm{S}, \mathrm{T}, \mathrm{P}$ and $\mathrm{Q}$ have a unique common fixed point in $\mathrm{X}$.

Proof: The proof follows from theorem 2.2, by considering the induced fuzzy metric space $(\mathrm{X}, \mathrm{M}, *)$ where $\mathrm{a} * \mathrm{~b}=\min \{\mathrm{a}, \mathrm{b}\}$ and $\mathrm{M}(\mathrm{x}, \mathrm{y}, \mathrm{t})=\frac{t}{t+d(x, y)}$. This result also generalizes the results of Pant and Jha[17], Balasubramaniam et .al .[1] and Kutukcu et .al .[12] for complete metric space in the aforesaid sense.

\section{REFERENCES}

[1] Balasubramaniam P, MuraliSankar S \& Pant R P, Common fixed points of four mappings in a fuzzy metric space, J.Fuzzy Math. 10(2)(2002), 379.

[2] Cho Y J, Pathak H K, Kang S M \& Jung J S, Common fixed points of compatible maps of type ( $\beta$ ) on fuzzy metric spaces, Fuzzy Sets and Systems 93(1998), 99.

[3] George A, Veeramani P, On some results in fuzzy metric spaces, Fuzzy Sets and Systems, 64(1994), 395.

[4] Grabiec M, Fixed points in fuzzy metric spaces, Fuzzy Sets and Systems 27(1988), 385.

[5] Hadzic O, Common fixed point theorems for families of mapping in complete metric space, Math.Japon. 29(1984), 127. 
[6] Jungck G, Compatible mappings and common fixed points(2), Internat.J.Math. Math. Sci.(1988), 285.

[7] Jungck G \& Rhodes B E, Fixed point for set valued functions without continuity, Indian J. Pure . appl . Math ., 29(3) (1998), 227.

[8] Kramosil O \& Michalek J, Fuzzy metric and statistical metric spaces, Kybernetica, 11(1975), 326.

[9] Kutukcu S, A fixed point theorem in manger spaces, Int.Math.F.1,32(2006), 1543.

[10] Kutukcu S, Yildiz C, Juna A, On common fixed points in Menger Probabilistic metric spaces, Int.J.Contemp.Math.Sci. 2(8)(2007), 383.

[11] Kutukcu S, A fixed point theorem for contraction type mappings in Menger Spaces. Am.J.Appl.Sci. 4(6)(2007), 371.

[12] Kutukcu S, Sharma $\mathrm{S} \&$ Tokgoz H, A fixed point theorem in fuzzy metric spaces. Int.J.Math.Analysis, 1(18)(2007), 861.

[13] Mishra S N, Common fixed points of Compatible mappings in PM-Spaces, Math.Japon.36(1991), 283.

[14] Mishra S N, Sharma N \& Singh S L, Common fixed Points of maps on fuzzy metric spaces, Internet.J.Math \& Math.Sci,17(1994), 253.

[15] Pant R P, Common fixed points of four mappings, Bull.Cal.Math.Soc. 90(1998), 281.

[16] Pant R P, Common fixed point theorems for contractive maps, J. Math. Anal. Appl. 226(1998), 251

[17] Pant R P \& Jha K, Aremarkon, Common fixed points of four mappings in a fuzzy metric space, J.Fuzzy Math. 12(2)(2004), 433.

[18] Rhoades B E, Contractive definitions and continuity, Contemporary Math.72(1988), 233.

[19] Sharma S, Common fixed point theorems in fuzzy metric spaces, Fuzzy Sets and Systems 127(2002), 345.

[20] Sharma S \& Deshpande B, Discontinuity and weak compatibility in fixed point consideration on non-complete fuzzy metric spaces, J. Fuzzy Math.11(2)(2003), 671.

[21] Sharma S \& Deshpande B, Compatible multivalued mappings satisfying an implicit relation, Southeast Asian. Bull.Math. 30(2006), 535. 
KATHMANDU UNIVERSITY JOURNAL OF SCIENCE, ENGINEERING AND TECHNOLOGY

VOL. 7, No. I, SEPTEMBER, 2011, pp 28- 37

[22] Vasuki R, Common fixed points for R-weakly commuting maps in fuzzy metric spaces, Indian J.Pure.Appl.Math.30(1999), 419.

[23] Zadeh L A, Fuzzy Sets, Information and Control, 8(1965) 338. 